\title{
Relation-Theoretic Contraction Principle in Cone Metric Spaces with Banach Algebra
}

\author{
S. K. Malhotra, J. B. Sharma, S. Shukla
}

\begin{abstract}
In this work, we introduce the notion of relation-theoretic contractions in cone metric spaces with Banach algebra and prove some fixed point results for such contractions. Our results generalize and unify several known results in the setting of cone metric spaces with Banach algebra. An example is provided which illustrate the results proved herein and shows that how the new results are different from existing ones.
\end{abstract}

Keywords: Cone metric space; Relation-theoretic contraction principle; Solid cone; Banach algebra; Fixed point.

\section{Introduction}

In 2003, Ran and Reurings [2] proved an analogue of Banach's fixed point theorem in partially ordered sets endowed with a metric. They showed that such results can be used in finding the solutions of linear and nonlinear matrix equations. Very recently, Alam and Imdad [1] introduced a novel variant of Banach's fixed point theorem on a complete metric space endowed with a binary relation which under universal relation reduces to Banach contraction principle. Further, they derived the results of Ran and Reurings [2], Nieto, R. Rodríguez-López [9, 10], Turinici [13, 14] from the relation-theoretic contraction principle.

On the other hand, Huang and Zhang [11] introduced the notion of cone metric spaces as a generalization of metric spaces. They proved an analogue of the Banach contraction principle in setting of cone metric spaces with the assumption that the cone is normal. Rezapour and Hamlbarani [18] showed that the assumption of normality of cone can be removed. Later, some authors (see, e.g., [6, 21, 22, 24]) showed that the fixed point results proved on cone metric spaces are the simple consequences of corresponding results of usual metric spaces.

Manuscript received October 21, 2015; accepted March 2, 2016.

S.K. Malhotra is with the Govt. Science \& Commerce College Benazeer, Bhopal, (M.P.) India; J.B. Sharma is with the Choithram College of Professional Studies, Dhar Road Indore, India; S. Shukla is with the Shri Vaishnav Institute of Technology \& Science Gram Baroli, Sanwer Road, Indore, India. 
In view of the arguments given in [6, 21, 22, 24], Liu and $\mathrm{Xu}$ [7] used the cones over a Banach algebra and proved some fixed point theorems on cone metric spaces. In the contractive condition used by Liu and Xu [7] on self-maps of cone metric spaces the contractive constant was replaced by a vector of cone. They also gave an example which shows that their fixed point results cannot be obtained by the corresponding results on usual metric spaces with an approach used, e.g., in [6, 21, 22, 24]. Although, Liu and Xu [7] used the normality of the underlying cone, when proving the fixed point results, but such fixed point results can be easily proved with non-normal cones, as shown by Xu and Radenović [17].

In this work, we prove the results of Alam and Imdad [1] in the setting of cone metric spaces with Banach algebra, and so, we generalize and unify the results of Alam and Imdad [1], Huang and Zhang [11], Liu and Xu [7] and several other results in cone metric spaces with Banach algebra. An example is provided which illustrate the results proved herein and shows that how the new results are different from existing ones.

\section{Preliminaries}

The following definitions can be found, e.g., in [19].

Let $A$ be a real Banach algebra, i.e., $A$ is a real Banach space in which an operation of multiplication is defined, subject to the following properties: for all $x, y, z \in A, a \in \mathbb{R}$

1. $x(y z)=(x y) z$

2. $x(y+z)=x y+x z$ and $(x+y) z=x z+y z$

3. $a(x y)=(a x) y=x(a y)$;

4. $\|x y\| \leq\|x\|\|y\|$.

In this paper, we shall assume that the Banach algebra $A$ has a unit, i.e., a multiplicative identity $e$ such that $e x=x e=x$ for all $x \in A$. An element $x \in A$ is said to be invertible if there is an inverse element $y \in A$ such that $x y=y x=e$. The inverse of $x \in X$ is denoted by $x^{-1}$. For more details we refer to [19].

The following proposition is well known [19].

Proposition 1. Let $A$ be a real Banach algebra with a unit e and $x \in A$. If the spectral radius $\rho(x)$ of $x$ is less than one, i.e.,

$$
\rho(x)=\lim _{n \rightarrow \infty}\left\|x^{n}\right\|^{\frac{1}{n}}=\inf _{n \geq 1}\left\|x^{n}\right\|^{\frac{1}{n}}<1
$$

then $e-x$ is invertible. Actually,

$$
(e-x)^{-1}=\sum_{i=0}^{\infty} x^{i}
$$


A subset $P$ of $A$ is called a cone if

1. $P$ is non-empty, closed and $\{\theta, e\} \subset P$, where $\theta$ is the zero vector of $A$;

2. $a_{1} P+a_{2} P \subset P$ for all non-negative real numbers $a_{1}, a_{2}$;

3. $P^{2}=P P \subset P$

4. $P \bigcap(-P)=\{\theta\}$.

For a given cone $P \subset A$, we can define a partial ordering $\preceq$ with respect to $P$ by $x \preceq y$ if and only if $y-x \in P$. The notation $x \ll y$ will stand for $y-x \in P^{\circ}$, where $P^{\circ}$ denotes the interior of $P$.

The cone $P$ is called normal if there exists a number $K>0$ such that for all $a, b \in A$,

$$
a \preceq b \text { implies }\|a\| \leq K\|b\| .
$$

The least positive value of $K$ satisfying the above inequality is called the normal constant (see [11]). Note that, for any normal cone $P$ we have $K \geq 1$ (see [18]). In the following we always assume that $P$ is a cone in a real Banach algebra $A$ with $P^{\circ} \neq \phi$ (i.e., the cone $P$ is a solid cone) and $\preceq$ is the partial ordering with respect to $P$.

The following lemmas and remarks will be useful in the sequel.

Lemma 1 (See [16, 23]). If $E$ is a real Banach space with a cone $P$ and:

(a) If $a \preceq \lambda a$ with $a \in P$ and $0 \leq \lambda<1$, then $a=\theta$.

(b) If $E$ is a real Banach space with a solid cone $P, u \in P$ and if $\theta \preceq u \ll c$ for each $\theta \ll c$, then $u=\theta$.

(c) If $E$ is a real Banach space with a solid cone $P$ and if $\left\|x_{n}\right\| \rightarrow 0$ as $n \rightarrow \infty$, then for any $\theta \ll c$, there exists $n_{0} \in \mathbb{N}$ such that, $x_{n} \ll c$ for all $n<n_{0}$.

Remark 2 (See [17]). If $\rho(a)<1$ then $\left\|a^{n}\right\| \rightarrow 0$ as $n \rightarrow \infty$.

Remark 3. If $a, b, c \in P$ and $a \ll b$, then $a c \preceq b c$.

Proof. Since $a \ll b$ we have $b-a \in P^{\circ}$. Again, as $c \in P$, by the definition of cone we have $c(b-a) \in P$, i.e., $c b-c a \in P$. Therefore, $a c \preceq a b$.

Definition 4 (See $[7,8,11]$ ). Let $X$ be a non-empty set. Suppose that the mapping $d: X \times$ $X \rightarrow$ A satisfies:

1. $\theta \preceq d(x, y)$ for all $x, y \in X$ and $d(x, y)=\theta$ if and only if $x=y$. 
2. $d(x, y)=d(y, x)$ for all $x, y \in X$.

3. $d(x, y) \preceq d(x, z)+d(z, y)$ for all $x, y, z \in X$.

Then $d$ is called a cone metric on $X$, and $(X, d)$ is called a cone metric space over the Banach algebra A.

Definition 5 (See [11]). Let $(X, d)$ be a cone metric space, $x \in X$ and $\left\{x_{n}\right\}$ be a sequence in $X$. Then:

1. The sequence $\left\{x_{n}\right\}$ converges to $x$ whenever for each $c \in A$ with $\theta \ll c$, there is $n_{0} \in \mathbb{N}$ such that $d\left(x_{n}, x\right) \ll$ c for all $n>n_{0}$. We denote this by $\lim _{n \rightarrow \infty} x_{n}=x$ or $x_{n} \rightarrow x$ as $n \rightarrow \infty$.

2. The sequence $\left\{x_{n}\right\}$ is a Cauchy sequence whenever for each $c \in A$ with $\theta \ll c$, there is $n_{0} \in \mathbb{N}$ such that $d\left(x_{n}, x_{m}\right) \ll c$ for all $n, m>n_{0}$.

3. $(X, d)$ is a complete cone metric space if every Cauchy sequence is convergent in $X$.

It is obvious that the limit of a convergent sequence in a cone metric space is unique. A mapping $T: X \rightarrow X$ is called continuous at $x \in X$, if for every sequence $\left\{x_{n}\right\}$ in $X$ such that $x_{n} \rightarrow x$ as $n \rightarrow \infty$, we have $T x_{n} \rightarrow T x$ as $n \rightarrow \infty$.

Next, we sate some definitions and properties about the relations.

Definition 6 (See [15]). Let $X$ be a nonempty set. A subset $\mathscr{R}$ of $X \times X$ is called a binary relation on $X$. Notice that for each pair $x, y \in X$ one of the following conditions holds:

(i) $(x, y) \in \mathscr{R}$ which amounts to saying that " $x$ is $\mathscr{R}$-related to $y$ " or " $x$ relates to $y$ under $\mathscr{R}$." Sometimes, we write $x \mathscr{R} y$ instead of $(x, y) \in \mathscr{R}$;

(ii) $(x, y) \notin \mathscr{R}$; which means that " $x$ is not $\mathscr{R}$-related to $y$ " or " $x$ does not relate to $y$ under $\mathscr{R}$.”

Trivially, $X \times X$ and $\emptyset$ being subsets of $X \times X$ are binary relations on $X$, which are respectively called the universal relation (or full relation) and empty relation. Another important relation of this kind is the relation

$$
\Delta_{X}=\{(x, x): x \in X\}
$$

called the identity relation or the diagonal relation on $X$. Throughout this paper, $\mathscr{R}$ stands for a nonempty binary relation, but for the sake of simplicity, we write only "binary relation" instead of "nonempty binary relation".

Definition 7 (See [1]). Let $\mathscr{R}$ be a binary relation defined on a nonempty set $X$ and $x, y \in X$. We say that $x$ and $y$ are $\mathscr{R}$-comparative if either $(x, y) \in \mathscr{R}$ or $(y, x) \in \mathscr{R}$. We denote it by $[x, y] \in \mathscr{R}$. 
Definition 8 (See [15]). Let $X$ be a nonempty set and $\mathscr{R}$ a binary relation on $X$.

(1) The inverse, transpose or dual relation of $\mathscr{R}$, denoted by $\mathscr{R}^{-1}$, is defined by $\mathscr{R}^{-1}=$ $\{(x, y) \in X \times X:(y, x) \in \mathscr{R}\}$.

(2) The reflexive closure of $\mathscr{R}$, denoted by $\mathscr{R}^{\#}$, is defined to be the set $\mathscr{R} \cup \Delta_{X}$ (i.e., $\mathscr{R}^{\#}$ : = $\mathscr{R} \cup \Delta_{X}$ ). Indeed, $\mathscr{R}^{\#}$ is the smallest reflexive relation on $X$ containing $\mathscr{R}$.

(3) The symmetric closure of $\mathscr{R}$, denoted by $\mathscr{R}^{\text {s }}$, is defined to be the set $\mathscr{R} \cup \mathscr{R}^{-1}$ (i.e., $\mathscr{R}^{s}:=\mathscr{R} \cup \mathscr{R}^{-1}$ ). Indeed, $\mathscr{R}^{s}$ is the smallest symmetric relation on $X$ containing $\mathscr{R}$.

Proposition 9 (See [1]). For a binary relation $\mathscr{R}$ defined on a nonempty set $X$,

$$
(x, y) \in \mathscr{R}^{s} \Longleftrightarrow[x, y] \in \mathscr{R} \text {. }
$$

Definition 10 (See [1]). Let $X$ be a nonempty set and $\mathscr{R}$ a binary relation on $X$. Then:

(a) A sequence $\left\{x_{n}\right\}$ in $X$ is called $\mathscr{R}$-preserving if $\left(x_{n}, x_{n+1}\right) \in \mathscr{R}$ for all $n \in \mathbb{N}$.

(b) A mapping $T: X \rightarrow X$ is called $T$-closed if for every $(x, y) \in \mathscr{R}$ we have $(T x, T y) \in \mathscr{R}$.

Proposition 11 (See [1]). Let X, T and $\mathscr{R}$ be the same as in Definition 10. If $\mathscr{R}$ is $T$-closed, then $\mathscr{R}^{s}$ is also $T$-closed.

Definition 12 (See [3, 4]). Let $X$ be a nonempty set and $\mathscr{R}$ a binary relation on $X$.

(a) A subset $E$ of $X$ is called $\mathscr{R}$-directed if for each $x, y \in E$, there exists $z \in X$ such that $(x, z) \in \mathscr{R}$ and $(y, z) \in \mathscr{R}$.

(b) For $x, y \in X$, a path of length $r$ (where $r$ is a natural number) in $\mathscr{R}$ from $x$ to $y$ is a finite sequence $\left\{z_{i}\right\}_{i=0}^{k} \subset X$ satisfying the following conditions:

(i) $z_{0}=x$ and $z_{r}=y$;

(ii) $\left(z_{i}, z_{i+1}\right) \in \mathscr{R}$ for each $0 \leq i \leq r-1$.

In this paper, following [1] we use the following notation:

(i) $F(T)=$ the set of all fixed points of $T$;

(ii) $X(T ; \mathscr{R}):=\{x \in X:(x, T x) \in \mathscr{R}\}$;

(iii) $\mathscr{Y}(x, y, \mathscr{R}):=$ the class of all paths in $\mathscr{R}$ from $x$ to $y$. 


\section{Main results}

First, we define the generalized relation-theoretic contractions on cone metric spaces and a proposition which will be used in the sequel.

Definition 13. Let $(X, d)$ be a complete cone metric space over a Banach algebra $A, P$ be the underlying solid cone and $\mathscr{R}$ be a binary relation on $X$. Then the mapping $T: X \rightarrow X$ is said to be generalized relation-theoretic contraction if there exists $k \in P$ such that $\rho(k)<1$ and,

$$
d(T x, T y) \preceq k d(x, y)
$$

for all $x, y \in X$ with $(x, y) \in \mathscr{R}$. The vector $k$ is called the contractive vector of $T$.

The proof of the following proposition is similar to that of Proposition 2.3 of [1], therefore, we omit the proof.

Proposition 14. Let $(X, d)$ be a cone metric space over a Banach algebra $A$ and, $P$ be the underlying solid cone and $\mathscr{R}$ be a binary relation on $X$. Then, for a mapping $T: X \rightarrow X$ and $k \in P$ the following generalized contractive conditions are equivalent:

(I) $d(T x, T y) \preceq k d(x, y)$ for all $x, y \in X$ with $(x, y) \in \mathscr{R}$;

(II) $d(T x, T y) \preceq k d(x, y)$ for all $x, y \in X$ with $[x, y] \in \mathscr{R}$.

Definition 15. Let $(X, d)$ be a cone metric space over a Banach algebra $A, P$ the underlying solid cone and $\mathscr{R}$ be a binary relation on $X$. Then, $\mathscr{R}$ is called $d$-self-closed if for every $\mathscr{R}$-preserving sequence $\left\{x_{n}\right\}$ with $x_{n} \rightarrow x \in X$ as $n \rightarrow \infty$, there exists a subsequence $\left\{x_{n_{k}}\right\}$ of $\left\{x_{n}\right\}$ such that $\left[x_{n_{k}}, x\right] \in \mathscr{R}$ for all $k \in \mathbb{N}$.

The following theorem is an existence result for a generalized relation-theoretic contraction on cone metric spaces over Banach algebra.

Theorem 16. Let $(X, d)$ be a complete cone metric space over a Banach algebra $A, P$ the underlying solid cone and $\mathscr{R}$ be a binary relation on $X$. Suppose, $T: X \rightarrow X$ be a generalized relation-theoretic contraction with contractive vector $k$ and the following conditions are satisfied:

(i) $X(T ; \mathscr{R})$ is nonempty;

(ii) $\mathscr{R}$ is T-closed;

(iii) either $T$ is continuous or $\mathscr{R}$ is $d$-self-closed.

Then $T$ has a fixed point $x^{*} \in X$. 
Proof. Because, $X(T ; \mathscr{R}) \neq \emptyset$, let $x_{0} \in X(T ; \mathscr{R})$, i.e., $\left(x_{0}, T x_{0}\right) \in \mathscr{R}$. Define a sequence $\left\{x_{n}\right\}$ by

$$
x_{n}=T x_{n-1} \text { for all } n \in \mathbb{N} .
$$

We shall show that the sequence $\left\{x_{n}\right\}$ is $\mathscr{R}$-preserving. Then, by definition we have $\left(x_{0}, x_{1}\right) \in$ $\mathscr{R}$. By $T$-closedness of $\mathscr{R}$ we obtain $\left(T x_{0}, T x_{1}\right)=\left(x_{1}, x_{2}\right) \in \mathscr{R}$. Repetition of this argument gives

$$
\left(x_{n-1}, x_{n}\right) \in \mathscr{R} \text { for all } n \in \mathbb{N} \text {. }
$$

Thus, the sequence $\left\{x_{n}\right\}$ is $\mathscr{R}$-preserving. Since $T$ is a generalized relation-theoretic contraction with contractive vector $k$ and the sequence $\left\{x_{n}\right\}$ is $\mathscr{R}$-preserving, we obtain; for all $n \in \mathbb{N}$

$$
d\left(x_{n}, x_{n+1}\right)=d\left(T x_{n-1}, T x_{n}\right) \preceq k d\left(x_{n-1}, x_{n}\right)
$$

which by induction yields that

$$
d\left(x_{n}, x_{n+1}\right) \preceq k^{n} d\left(x_{0}, x_{1}\right) \text { for all } n \in \mathbb{N} .
$$

Thus, for $n<m$ we have

$$
\begin{aligned}
d\left(x_{n}, x_{m}\right) & \preceq d\left(x_{n}, x_{n+1}\right)+d\left(x_{n+1}, x_{n+2}\right)+\cdots+d\left(x_{m-1}, x_{m}\right) \\
& \preceq k^{n} d\left(x_{0}, x_{1}\right)+k^{n+1} d\left(x_{0}, x_{1}\right)+\cdots+k^{m-1} d\left(x_{0}, x_{1}\right) \\
& =\left(e+k+\cdots+k^{m-n-1}\right) k^{n} d\left(x_{0}, x_{1}\right) \\
& \preceq\left(\sum_{i=0}^{\infty} k^{i}\right) k^{n} d\left(x_{0}, x_{1}\right) \\
& =(e-k)^{-1} k^{n} d\left(x_{0}, x_{1}\right) .
\end{aligned}
$$

Since $\rho(k)<1$, by Remark 2 we have $\left\|k^{n}\right\| \rightarrow 0$ as $n \rightarrow \infty$. Therefore, by Lemma 1 it follows that: for every $c \in A$ with $\theta \ll c$ there exists $n_{0} \in \mathbb{N}$ such that

$$
d\left(x_{n}, x_{m}\right) \preceq(e-k)^{-1} k^{n} d\left(x_{0}, x_{1}\right) \ll c
$$

for all $n>n_{0}$. It implies that $\left\{x_{n}\right\}$ is a Cauchy sequence. By completeness of $X$, there exists $x^{*} \in X$ such that $x_{n} \rightarrow x^{*}$ as $n \rightarrow \infty$. We shall show that $x^{*}$ is a fixed point of $T$.

In view of (iii), we consider the following two cases:

Case I: Suppose $T$ is continuous. Then, it follows that $x_{n+1}=T x_{n} \rightarrow T x^{*}$ as $n \rightarrow \infty$. By the uniqueness of limit we get $x^{*}=T x^{*}$, that is $x^{*}$ is a fixed point of $T$.

Case I: Suppose $\mathscr{R}$ is $d$-self-closed. Then, since $\left\{x_{n}\right\}$ is $\mathscr{R}$-preserving and $x_{n} \rightarrow x$ as $n \rightarrow \infty$ by $d$-self-closedness of $\mathscr{R}$ there exists a subsequence $\left\{x_{n_{j}}\right\}$ of $\left\{x_{n}\right\}$ such that

$$
\left[x_{n_{j}}, x\right] \in \mathscr{R} \text { for all } j \in \mathbb{N} .
$$


Since $T$ is a generalized relation-theoretic contraction with contractive vector $k$, using Proposition 14 we obtain

$$
\begin{aligned}
d\left(x^{*}, T x^{*}\right) & \preceq d\left(x^{*}, x_{n_{j}+1}\right)+d\left(x_{n_{j}+1}, T x^{*}\right) \\
& =d\left(x^{*}, x_{n_{j}+1}\right)+d\left(T x_{n_{j}}, T x^{*}\right) \\
& \preceq d\left(x^{*}, x_{n_{j}+1}\right)+k d\left(x_{n_{j}}, x^{*}\right) .
\end{aligned}
$$

As $x_{n} \rightarrow x^{*}$ as $n \rightarrow \infty$, for every $c \in P$ with $\theta \ll c$ and for every $m \in \mathbb{N}$ there exists $j(m)$ such that $d\left(x_{n_{j}}, x^{*}\right) \ll \frac{c}{m}$ for all $j>j(m)$. Therefore, by Remark 3 we have $k d\left(x_{n_{j}}, x^{*}\right) \preceq \frac{k c}{m}$ and it follows from the above inequality that

$$
d\left(x^{*}, T x^{*}\right) \preceq \frac{c}{m}+\frac{k c}{m}=\frac{c}{m}(e+k) \text { for all } j>j(m), m \in \mathbb{N} .
$$

It implies that $\frac{c}{m}(e+k)-d\left(x^{*}, T x^{*}\right) \in P$ for all $m \in \mathbb{N}$. Since $P$ is closed, letting $m \rightarrow \infty$ we obtain $\theta-d\left(x^{*}, T x^{*}\right) \in P$. By definition, we must have $d\left(x^{*}, T x^{*}\right)=\theta$, i.e., $T x^{*}=x^{*}$. Thus, $x^{*}$ is a fixed point of $T$.

The above theorem ensures only the existence of fixed point of a generalized relationtheoretic contraction but not the uniqueness of fixed point (see Example 18 of this paper). The uniqueness of fixed point is considered in the following theorem.

Theorem 17. Let all the conditions of Theorem 16 are satisfied. In addition, suppose that the set $\mathscr{Y}\left(x, y, \mathscr{R}^{s}\right)$ is nonempty for each $x, y \in X$, then $T$ has a unique fixed point $x^{*} \in X$.

Proof. Following similar arguments to those in the proof of Theorem 16 we obtain the existence of fixed point $x^{*} \in X$. Let the set $\mathscr{Y}\left(x, y, \mathscr{R}^{s}\right)$ is nonempty for each $x, y \in X$. We shall show that $x^{*}$ is the unique fixed point of $T$. On contrary, suppose that $y^{*} \in F(T)$ and $x^{*} \neq y^{*}$, i.e.,

$$
x^{*}=T x^{*} \neq y^{*}=T y^{*} .
$$

Then by assumption there exists a path $\left\{z_{i}\right\}_{i=0}^{r}$ of length $r$ in $\mathscr{R}^{s}$ such that

$$
z_{0}=x^{*}, z_{r}=y^{*},\left[z_{i}, z_{i+1}\right] \in \mathscr{R} \text { for } i=0,1, \ldots, r-1 .
$$

As $\mathscr{R}$ is $T$-closed, using Proposition 11 we have $\mathscr{R}^{s}$ is also $T$-closed, and so

$$
\left[T^{n} z_{i}, T^{n} z_{i+1}\right] \in \mathscr{R} \text { for } i=0,1, \ldots, r-1 \text { and for all } n \geq 0 \text {. }
$$


Now, using Proposition 14, (2), (3) and (4) we obtain

$$
\begin{aligned}
d\left(x^{*}, y^{*}\right) & =d\left(T^{n} x^{*}, T^{n} y^{*}\right)=d\left(T^{n} z_{0}, T^{n} z_{r}\right) \\
& \preceq \sum_{i=0}^{r-1} d\left(T^{n} z_{i}, T^{n} z_{i+1}\right) \\
& \preceq k \sum_{i=0}^{r-1} d\left(T^{n-1} z_{i}, T^{n-1} z_{i+1}\right) \\
& \preceq k^{2} \sum_{i=0}^{r-1} d\left(T^{n-2} z_{i}, T^{n-2} z_{i+1}\right) \\
& \preceq \cdots \preceq k^{n} \sum_{i=0}^{r-1} d\left(z_{i}, z_{i+1}\right) \text { for all } n \in \mathbb{N} .
\end{aligned}
$$

Since $\rho(k)<1$, by Remark 2 we have $\left\|k^{n}\right\| \rightarrow 0$ as $n \rightarrow \infty$, and so,

$$
\left\|k^{n} \sum_{i=0}^{r-1} d\left(z_{i}, z_{i+1}\right)\right\| \leq\left\|k^{n}\right\|\left\|\sum_{i=0}^{r-1} d\left(z_{i}, z_{i+1}\right)\right\| \rightarrow 0 \text { as } n \rightarrow \infty .
$$

Therefore, by Lemma 1 it follows that: for every $c \in A$ with $\theta \ll c$ there exists $n_{0} \in \mathbb{N}$ such that

$$
d\left(x^{*}, y^{*}\right) \preceq k^{n} \sum_{i=0}^{r-1} d\left(z_{i}, z_{i+1}\right) \ll c .
$$

It implies that $d\left(x^{*}, y^{*}\right)=\theta$, i.e., $x^{*}=y^{*}$. This contradiction shows that the fixed point is unique.

Next, we give an example which illustrate the above result.

Example 18. Let $A=C_{\mathbb{R}}^{1}[0,1] \times C_{\mathbb{R}}^{1}[0,1]$ with the norm

$$
\left\|\left(x_{1}, x_{2}\right)\right\|=\left\|x_{1}\right\|_{\infty}+\left\|x_{2}\right\|_{\infty}+\left\|x_{1}^{\prime}\right\|_{\infty}+\left\|x_{2}^{\prime}\right\|_{\infty} .
$$

Define the multiplication on A by

$$
x y=\left(x_{1} y_{1}, x_{1} y_{2}+x_{2} y_{1}\right) \text { for all } x=\left(x_{1}, x_{2}\right), y=\left(y_{1}, y_{2}\right) \in A .
$$

Then, $A$ is a Banach algebra with usual sum of functions and scalar product on cartesian product $C_{\mathbb{R}}^{1}[0,1] \times C_{\mathbb{R}}^{1}[0,1]$ and with unit e $=(1,0)$. Let $P=\left\{\left(x_{1}(t), x_{2}(t)\right) \in A: x_{1}(t), x_{2}(t) \geq\right.$ $0, t \in[0,1]\}$. Then $P$ is a solid cone which is not normal.

Let $X=\mathbb{R}^{+} \times \mathbb{R}^{+}, \mathbb{R}^{+}=[0, \infty)$ and define the cone metric $d: X \times X \rightarrow P$ by

$$
d\left(\left(x_{1}, x_{2}\right),\left(y_{1}, y_{2}\right)\right)=\left(\left|x_{1}-y_{1}\right|,\left|x_{2}-y_{2}\right|\right) e^{t} \in P .
$$


Then, $(X, d)$ is a complete cone metric space. By $S_{\mathbb{Q}^{+}}^{0}$ we denote the set of all sequences of nonnegative rational numbers converging to zero. Obviously, $S_{\mathbb{Q}^{+}}^{0} \neq \emptyset$. For a positive rational number $a$, define the mapping $T: X \rightarrow X$ and the binary relation $\mathscr{R}$ on $X$ by:

$$
T\left(x_{1}, x_{2}\right)= \begin{cases}\left(\frac{x_{1}}{2}, \frac{x_{2}}{2}+a x_{1}\right), & \text { if } x_{1}, x_{2} \in \mathbb{Q}^{+} \\ \left(x_{1}\left|x_{1}^{2}-1\right|, x_{2}\left|x_{2}^{2}-1\right|\right), & \text { otherwise }\end{cases}
$$

and

$$
\mathscr{R}=\left\{\left(\left(x_{n}, y_{n}\right),\left(z_{n}, u_{n}\right)\right), n \in \mathbb{N}:\left\{x_{n}\right\},\left\{y_{n}\right\},\left\{z_{n}\right\},\left\{u_{n}\right\} \in S_{\mathbb{Q}^{+}}^{0}\right\} .
$$

Then, $T$ is a generalized relation-theoretic contraction with contractive vector $k(t)=\left(k_{1}, k_{2}\right)$, where $k_{1} \in\left[\frac{1}{2}, 1\right)$ and $k_{2} \in[a, \infty), \rho(k)=k_{1}$. Indeed, $\left(\left(x_{n}, y_{n}\right),\left(z_{n}, u_{n}\right)\right) \in \mathscr{R}$ implies that $x_{n}, y_{n}, z_{n}, u_{n} \in \mathbb{Q}^{+}$. Therefore,

$$
\begin{aligned}
d\left(T\left(x_{n}, y_{n}\right), T\left(z_{n}, u_{n}\right)\right) & =\left(\frac{1}{2}\left|x_{n}-z_{n}\right|,\left|\frac{1}{2}\left(y_{n}-u_{n}\right)+a\left(x_{n}-z_{n}\right)\right|\right) e^{t} \\
& \preceq\left(k_{1}\left|x_{n}-z_{n}\right|, k_{1}\left|y_{n}-u_{n}\right|+k_{2}\left|x_{n}-z_{n}\right|\right) e^{t} \\
& =\left(k_{1}, k_{2}\right)\left(\left|x_{n}-z_{n}\right|,\left|y_{n}-u_{n}\right|\right) e^{t} \\
& =k d\left(\left(x_{n}, y_{n}\right),\left(z_{n}, u_{n}\right)\right) .
\end{aligned}
$$

Since $S_{\mathbb{Q}^{+}}^{0} \neq \emptyset$, let $\left\{x_{n}\right\} \in S_{\mathbb{Q}^{+}}^{0}$. Then, as a is positive rational, we have

$$
\left(\left(x_{n}, x_{n}\right), T\left(x_{n}, x_{n}\right)\right)=\left(\left(x_{n}, x_{n}\right),\left(\frac{x_{n}}{2}, \frac{x_{n}}{2}+a x_{n}\right)\right) \in \mathscr{R} .
$$

Therefore, $X(T ; \mathscr{R}) \neq \emptyset$.

Again, for every $\left(\left(x_{n}, y_{n}\right),\left(z_{n}, u_{n}\right)\right) \in \mathscr{R}$ we have $\left\{x_{n}\right\},\left\{y_{n}\right\},\left\{z_{n}\right\},\left\{u_{n}\right\} \in S_{\mathbb{Q}^{+}}^{0}$, and so,

$$
\left(T\left(x_{n}, y_{n}\right), T\left(z_{n}, u_{n}\right)\right)=\left(\left(\frac{x_{n}}{2}, \frac{y_{n}}{2}+a x_{n}\right),\left(\frac{z_{n}}{2}, \frac{u_{n}}{2}+a z_{n}\right)\right) \in \mathscr{R} .
$$

Therefore, $\mathscr{R}$ is $T$-closed. Finally, if $\left\{\left(x_{n}, y_{n}\right)\right\}$ is a sequence in $X$ such that

$$
\left(\left(x_{n}, y_{n}\right),\left(x_{n+1}, y_{n+1}\right)\right) \in \mathscr{R},\left(x_{n}, y_{n}\right) \rightarrow(x, y) \text { as } n \rightarrow \infty
$$

then we have $\left\{x_{n}\right\},\left\{y_{n}\right\} \in S_{\mathbb{Q}^{+}}^{0}$, and so, $(x, y)=(0,0)$. Therefore, by definition of $\mathscr{R}$ we have

$$
\left(\left(x_{n}, y_{n}\right),(x, y)\right) \in \mathscr{R} \text { for all } n \in \mathbb{N} \text {. }
$$

Therefore, $\mathscr{R}$ is $d$-self-closed. Thus, all the properties of Theorem 16 are satisfied and we conclude that there exists a fixed point of the mapping $T$. Indeed, $T$ has four fixed points in $X$ and

$$
F(T)=\{(0,0),(\sqrt{2}, 0),(0, \sqrt{2}),(\sqrt{2}, \sqrt{2})\} .
$$


Remark 19. In the above example, one can take $k=\left(\frac{1}{2}, a\right)$, and then the mapping $T$ is not continuous. Also, $\left(\frac{1}{2}, a\right) \npreceq(1,0)=e$ and $\left\|\left(\frac{1}{2}, a\right)\right\|=\frac{1+2 a}{2}>1$ (for $a>1$ ). For large enough a one can see that the mapping is not a Relation-theoretic contraction in the sense of Alam and Imdad [1] with respect to Euclidian metric on X. Again, since the fixed point of $T$ is not unique, the mapping $T$ is not a contraction in the sense of Liu and Xu [7]. Therefore, these known results are not applicable on the mapping T. Moreover, following similar arguments to those in the Remark 2.3 of the paper [7] we can say that our results are actual generalization of the known results.

\section{Consequences}

In this section, we derive the cone metric version of several existing results in the literature.

The following theorem improves the Theorem 2.1 of Liu and Xu [7] in cone metric spaces when the underlying cone is not necessarily normal.

Theorem 20 (Xu and Radenović [17]). Let $(X, d)$ be a complete cone metric space over a Banach algebra $A$ and $P$ be the underlying solid cone with $k \in P$ where $\rho(k)<1$. Suppose the mapping $T: X \rightarrow X$ satisfies generalized Lipschitz condition :

$$
d(T x, T y) \preceq k d(x, y) \text { for all } x, y \in X .
$$

Then $T$ has a unique fixed point in X. Moreover, for any $x \in X$, the iterative sequence $\left\{T^{n} x\right\}$ converges to the fixed point of $X$.

Proof. Define the relation $\mathscr{R}$ on $X$ by $\mathscr{R}=X \times X$, i.e., the universal relation on $X$. Then, all the conditions of Theorem 17 are satisfied, and so, the mapping $T$ has a unique fixed point in $X$.

The following theorem is a variant and the cone metric version of the main result of Ran and Reurings [2].

Theorem 21. Let $(X, \sqsubseteq)$ be a partially ordered set and suppose that $(X, d)$ be a complete cone metric space over a Banach algebra A with $P$ the underlying solid cone. Let $T: X \rightarrow X$ be continuous, monotone (i.e., either order-preserving or order-reversing) with respect to $\sqsubseteq$. Suppose that the following two assumptions hold:

(i) there exists $k \in P$ such that $\rho(k)<1$ and $d(T x, T y) \preceq k d(x, y)$ for all $x, y \in X$ with $x \sqsubseteq y$;

(ii) there exists $x_{0} \in X$ such that $x_{0} \sqsubseteq T x_{0}$ or $T x_{0} \sqsubseteq x_{0}$.

Then, $T$ has a fixed point in $X$. 
Proof. Define the relation $\mathscr{R}_{O}$ on $X$ by

$$
\mathscr{R}_{O}=\{(x, y) \in X \times X: x \sqsubseteq y\} .
$$

Then by definition

$$
\mathscr{R}_{O}^{-1}=\{(x, y) \in X \times X: y \sqsubseteq x\} .
$$

Now consider the symmetric relation $\mathscr{R}_{O}^{s}=\mathscr{R}_{O} \cup \mathscr{R}_{O}^{-1}$. Note that, the condition (i) implies that the mapping $T$ a generalized relation-theoretic contraction with contractive vector $k$, where $\rho(k)<1$. Since $T$ is monotone, we have $\mathscr{R}_{O}^{s}$ is $T$-closed. The condition (ii) implies that, there exists $x_{0} \in X$ such that $\left(x_{0}, T x_{0}\right) \in \mathscr{R}_{O}^{s}$. Therefore, $X\left(T ; \mathscr{R}_{O}^{s}\right)$ is nonempty. Finally, since $T$ is continuous, so all the conditions of Theorem 16 (with the relation $\mathscr{R}_{O}^{s}$ ) are satisfied, and so, the mapping $T$ has a fixed point in $X$.

The following theorem is a variant and the cone metric version of Nieto and RodríguezLópez $[9,10]$ when the cone metric is endowed with a Banach algebra.

Theorem 22. Let $(X, \sqsubseteq)$ be a partially ordered set and suppose that $(X, d)$ be a complete cone metric space over a Banach algebra A with $P$ the underlying solid cone. Let $T: X \rightarrow X$ be a monotone (nondecreasing or nonincreasing) mapping with respect to $\sqsubseteq$. Suppose that the following three assumptions hold:

(i) there exists $k \in P$ such that $\rho(k)<1$ and $d(T x, T y) \preceq k d(x, y)$ for all $x, y \in X$ with $y \sqsubseteq x ;$

(ii) there exists $x_{0} \in X$ such that $x_{0} \sqsubseteq T x_{0}$ or $T x_{0} \sqsubseteq x_{0}$;

(iii) if $\left\{x_{n}\right\}$ is a sequence in $X$ whose consecutive terms are comparable and $x_{n} \rightarrow x \in X$ as $n \rightarrow \infty$, then there exists a subsequence $\left\{x_{n_{k}}\right\}$ of $\left\{x_{n}\right\}$ such that every term of $\left\{x_{n_{k}}\right\}$ is comparable to the limit $x$.

Then, $T$ has a fixed point in $X$.

Proof. Let the relations $\mathscr{R}_{O}$ and $\mathscr{R}_{O}^{s}$ be similar to those in the proof of Theorem 21. Then again, all the conditions of Theorem 16 are satisfied (with the relation $\mathscr{R}_{O}^{s}$ ) and the result follows.

Remark 23. In a similar manner, we find the following:

- A relation $\mathscr{R}$ on a nonempty set $X$ is called a preorder if $\mathscr{R}$ is reflexive (i.e., $(x, x) \in \mathscr{R}$ for all $x \in X)$ and transitive (i.e., $(x, y),(y, z) \in \mathscr{R} \Longrightarrow(x, z) \in \mathscr{R})$. Now, take $\mathscr{R}$ as a preorder in Theorem 17, we obtain the cone metric version of Theorem 1 of Turinici [12].

- Take $\mathscr{R}$ as a transitive relation in Theorem 17 , we obtain a natural cone metric versions of Theorems 2.2 and 2.4 of Ben-El-Mechaiekh [5]. 


\section{References}

[1] A. Alam, M. Imdad, Relation-theoretic contraction principle, J. Fixed Point Theory Appl., 17(4) (2015) 693-702.

[2] A.C.M. RAN, M.C.B. REURINGS,A fixed point theorem in partially ordered sets and some applications to matrix equations, Proc. Amer. Math. Soc., 132 (2003) 1435-1443.

[3] B. Kolman, R.C. Busby, S. Ross,Discrete Mathematical Structures, 3rd ed., PHI Pvt. Ltd., New Delhi, 2000.

[4] B. SAmet, M. TuRinici, Fixed point theorems on a metric space endowed with an arbitrary binary relation and applications, Commun. Math. Anal., 13 (2012), 82-97.

[5] H. BEN-EL-MeCHAIEKH, The RanReurings fixed point theorem without partial order: A simple proof, J. Fixed Point Theory Appl., 16 (2014), 373-383.

[6] H. ÇAKAlli, A. SönmeZ, Ç. GEnÇ, On an equivalence of topological vector space valued cone metric spaces and metric spaces, Appl. Math. Lett., 25, (2012) 429-433.

[7] H. LIU AND S.-Y. XU, Cone metric spaces with Banach algebras and fixed point theorems of generalized Lipschitz mappings, Fixed Point Theory Appl., 2013, 2013:320.

[8] H. LIU AND S.-Y. XU, Fixed point theorems of quasi-contractions on cone metric spaces with Banach algebras, Abstarct and Applied Analysis, Volume 2013, Article ID 187348, 5 pages.

[9] J.J. NiETo, R. RodRígueZ-LóPez, Contractive mapping theorems in partially ordered sets and applications to ordinary differential equations, Order 22 (2005) 223-239.

[10] J.J. NIETO, R. RODRÍGUEZ-LóPEZ, Existence and uniqueness of fixed point in partially oredered sets and applications to ordinary differential equations, Acta Math. Sin. (Engl. Ser.) 23 (2007) 2205-2212.

[11] L.-G. HUANG AND X. ZHANG, Cone metric spaces and fixed point theorems of contractive mappings, J. Math. Anal. Appl., 332 (2007) 1468-1476.

[12] M. TURINICI, Linear contractions in product ordered metric spaces, Ann. Univ. Ferrara Sez., VII Sci. Mat. 59 (2013) 187-198.

[13] M. TURINICI, Ran-Reurings fixed point results in ordered metric space, Libertas Math., 31 (2011) 49-55.

[14] M. TURINICI, Nieto-Lopez theorems in ordered metric space, Math. Student, 81 (2012) 219-229.

[15] S. LiPSCHUTZ, Schaum's Outlines of Theory and Problems of Set Theory and Related Topics, McGrawHill, New York, 1964.

[16] S. RADENOVIĆ, B.E. RHOADES, Fixed point theorem for two non-self mappings in cone metric spaces, Comput. Math. Appl., 57, 1701-1707 (2009)

[17] S. XU, S. RADENOVIĆ, Fixed point theorems of generalized Lipschitz mappings on cone metric spaces over Banach algebras without assumption of normality, Fixed Point Theory Appl., 2014, 2014:102. 
[18] Sh. Rezapour, R. HAmlbarani, Some notes on the paper Cone metric spaces and fixed point theorems of contractive mappings, Math. Anal. Appl., 345 (2008), 719-724.

[19] W. Rudin, Functional Analysis, 2nd ed., McGraw-Hill, 1991.

[20] W.A. Kirk, P.S. SRinivasan, P. VeEramani, Fixed points for mappings satisfying cyclical contractive conditions, Fixed Point Theory 4(1)(2003), 79-89.

[21] W.S. Du, A note on cone metric fixed point theory and its equivalence, Nonlinear Anal., 72(5), (2010) 2259-2261.

[22] Y. Feng, W. MaO, The equivalence of cone metric spaces and metric spaces, Fixed Point Theory, 11(2), (2010) 259-264.

[23] Z. Kadelburg, M. PaVlović, S. Radenović, Common fixed point theorems for ordered contractions and quasi-contractions in ordered cone metric spaces, Comput. Math. Appl. 59, 3148-3159 (2010)

[24] Z. Kadelburg, S. Radenović, V. RAKočEvić, A note on the equivalence of some metric and cone metric fixed point results, Appl. Math. Lett., 24, (2011) 370-374. 